\title{
Power Electronic Converters in Energy Harvesting Systems- A Review
}

\author{
Mr Vishnu R Nedungadi ${ }^{1}$ and Dr R Vidhyapriya ${ }^{2}$ \\ \{vishnunedungadi@gmail.com ${ }^{1}$, rvp.bme@psgtech.ac.in ${ }^{2}$ \} \\ Research Scholar, Dept. of Biomedical Engineering, PSG College of Technology, Tamil Nadu, \\ India ${ }^{1}$, Professor \& Head, Dept of Biomedical Engineering, PSG College of Technology, Tamil Nadu, \\ India $^{2}$
}

\begin{abstract}
The technical advancements made in the modern wireless sensor networks has enabled the realisation of low power modules incorporating various sensors. These developments have pushed the researchers to look at harvesting energy from the ambient environment. The reduced dependence on battery, its recharging or replacement bodes well for the environment. The energy harvesting modules can directly power only a few devices and that too only if they have very low power requirement. The typical energy harvesting module utilises dc-dc converters or rectifier-multiplier modules to achieve appropriate levels of output voltage. This paper provides a review on various power electronic converters used in such energy harvesting applications.
\end{abstract}

Keywords: Energy Harvesting, DC-DC converter, AC-DC Converters.

\section{Introduction}

The technique of capturing energy from the ambient environment has become more popular recently, mainly due to the acceptance of low power sensor integrated wearable devices like Mi Band series, FitBit, GoQii etc. The possibility of integrating these modules with energy harvesters improves the battery life of these devices. Also, few modules have been developed in which the power is directly delivered to the sensors without using an energy storage element. The size of these harvesters is usually very small and can be easily accommodated.

The energy harvesters are transducers that converts mechanical energy to electrical energy. The voltage output directly available from an energy harvester is small. The amount of energy generated is increased by using array of transducers in conjunction with power electronic converters [1-4].The different sources from which energy is harvested from the ambient environments is as listed out in table I. the minimum power that can be acquired form them is also listed in the same. The different transducers used for realising these techniques are piezoelectric transducers to convert mechanical energy into electrical technology [5-7], thermoelectric generators to convert body heat into equivalent electrical voltage, and RF antennae to capture the electrical energy in RF waves [8]. 
TABLE I.Different Sources for Ambient Energy Harvesting (EH) with Minimum Power that can be acquired

\begin{tabular}{|l|l|}
\hline Source for Energy Harvesting & Acquired Power \\
\hline Radio Frequency & $10 \mathrm{uW}$ \\
\hline Mechanical Vibrations or movements & $3.35 \mathrm{~mW}$ \\
\hline From Body Heat & $60 \mathrm{uW}$ \\
\hline Wind & $20 \mathrm{~mW}$ \\
\hline
\end{tabular}

Figure 1 shows a block diagram of a typical EH system. In this system, the harvester captures the energy from the surroundings. Since the voltage generated is usually too small, it is forwarded to a boost converter. Once the voltage is raised to a required level, it is given to the load.

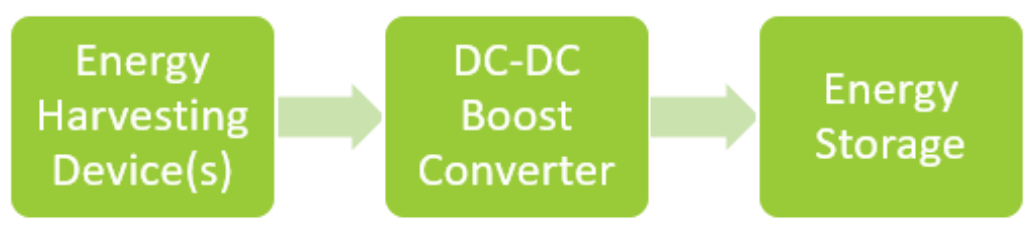

Fig 1. Block Diagram of a Typical Energy Harvesting System

The Wireless Sensor Networks (WSNs) are commonly powered using electromagnetic batteries. They have become increasingly popular due to their usage in monitoring devices. The significance of staying $\mathrm{ON}$ for as long as possible is one of important requirements of such devices. Hence, they have batteries as their main source of power. They have to be either recharged or replaced. Also, their proper disposal and recycling has become a huge challenge $[8,9]$. Since the common battery has a very short life span it is not opted for devices that are expected to have life span running in the range of 3-10 years[10,11]. The potential of EH as a solution to such problems is huge. It is relied upon to power electronic devices that are implanted in living beings, installed inside assets or placed in orbiting satellites. In section 2 converters and its classification is discussed. In section 3 the converters and its application in EH systems are discussed followed by conclusion as section 4 .

\section{Converters And Its Classification}

The electronic converters topology is classified into four categories, namely, AC-AC, AC-DC, DC-DC, and DC-AC [12,13]. The AC-DC converters are known as rectifiers and they convert $\mathrm{AC}$ waveformsinto $\mathrm{DC}$, which is suitable for $\mathrm{EH}$ applications. The basic application of DC-DC converters is to regulate the output voltage and maintain it at the required value in spite of any disturbances at its input.In EH applications, the output voltage from the transducers is very small. They have to be pulled up using DC-DC boost converters to appropriate levels before they can be utilised [24,25]. These are used mostly in vibration, wind, solar PV EH applications. The DC to AC converter is utilized to convert DC signal to $\mathrm{AC}$ waveform, which is suitable for thermoelectric $\mathrm{EH}$ applications. 
$A C-D C$ Converter: AC-DC converters or rectifiers,converts the $\mathrm{AC}$ waveforms to a constant DC output. It is possible to realise these converters at any point in the power spectrum. Uncontrolled rectifiers, also known as diode rectifiers or unidirectional AC-DC converters, consist of diodes. Hence it faces the inherent drawback of low power conversion efficiency and distortion. A filter is required to reduce the distortion or ripple. Figure 2 shows an AC-DC converter with multi-input, bridgeless resonantcircuit [14].

The conversion of low amplitude ac voltages from multiple sources to a controlled DC output voltage is realised with the help of the converter shown above. Various individual sources can be interfaced without using a diode bridge using this topology.Also, it is possible

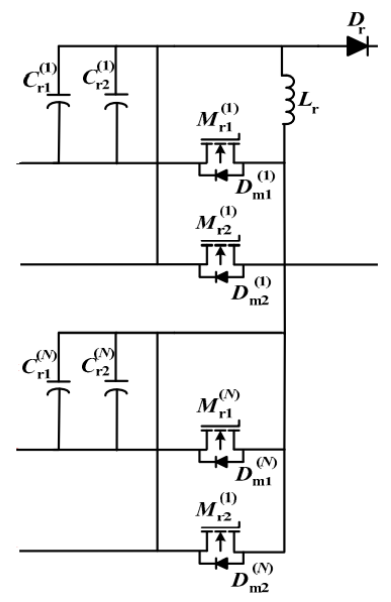

Fig 2. Multi Input Bridgeless Resonant Circuit

to pull up the output voltage appropriate levels. The control of multiple inductive sources can be realised with this topology. If $\mathrm{N}$ is the number assigned to each inductive source, then aSchottky diode, an inductor (resonant), and some MOSFET-capacitorbridges forms the multi-input circuit. All the input sourcesareconnected to one of the two MOSFET-capacitorbridges while sharing a resonant inductor with the Schottky diode.

$D C-D C$ Converter: The unidirectional DC-DC converter is one of the most commonly used converters in $\mathrm{EH}$ applications. The output voltage from these types of converters is maintained at a constant value irrespective of changes in the input. However, the open loop configuration of these converters have very weak regulation capability and the output voltage is affected whenever the circuit is subjected to any kind of disturbances. This can be addressed using the closed loop feedback topology withconventional controllers. The only controlling parameter directly available for modification is the duty ration of the semiconductor switch. Using PWM techniques, high efficiency closed loop converters can be realised for $\mathrm{EH}$ applications $[15,16]$. The DC-DC converters are divided intonon-isolated and isolatedconverters. The non-isolated topology consists of boost, buck, buck-boost, and Cuk converter. They consist of diode, inductors, capacitors, and a switch as shown in figure 3. 

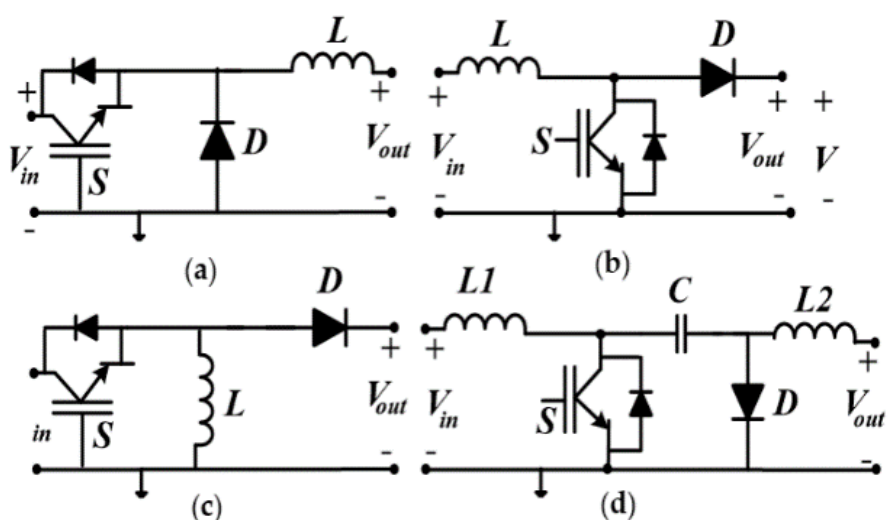

Fig 3.Non-isolated DC-DC converters: (a) buck, (b) boost, (c) buck- boost, and (d) Cuk

The isolated topologies consist of forward, flyback, and full-bridge converter and contains either a transformer or a coupled inductor. The topologies are as shown in figure 4.

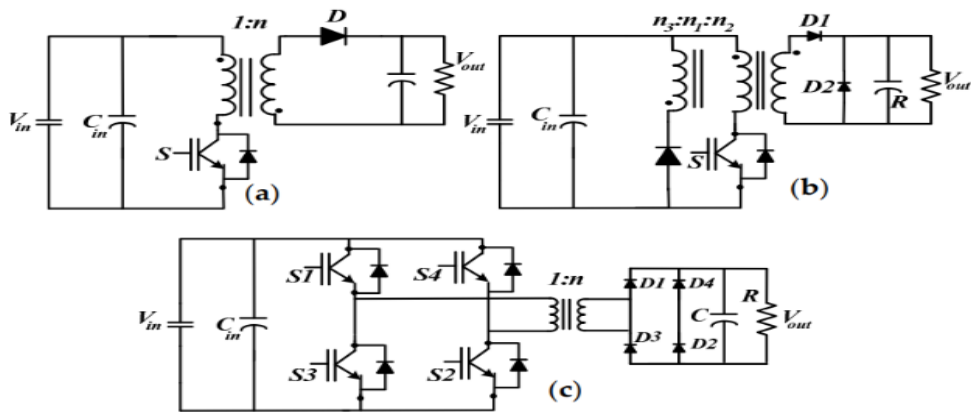

Fig 4. Isolated DC-DC converter: (a) flyback converter, (b) forward converter, and (c) full-bridge converter

The buck converter steps down the input voltage, i.e, the output voltage will be less than that of the input. The boost converter, as the name suggests, steps up the output voltage and maintains it at the required value. The buck-boost converter is similar to the boost converter. It is a hybrid version of the buck and boost topologies. When the duty cycle is set to a value between 0 and 0.5 , the circuit operates in the buck mode and in the boost mode when the duty cycle valueis set between 0.5 and 1 . It was understood from the literature that the boost and buck-boost topologies are often preferred in the energy harvesting applications.

A variation of the boost converter, with the switched inductor has also been used in the energy harvesting applications. Here the converter takes advantage of a MOSFET's capability of bidirectional current conduction. Dual capacitor topologies are preferred in these applications due to the low operation frequency [17,27]. This helps to suppress the ripple 
content in the output voltage to a great extent. Table II shows a comparison of various converter circuits used in the energy harvesting systems.

TABLE II. Comparison of Various Converter Circuits Used in Energy Harvesting Systems

\begin{tabular}{|l|l|l|}
\hline Converter & Complexity & Efficiency (\%) \\
\hline Buck & Low & $55-70$ \\
\hline Boost & Low & $70<$ \\
\hline Buck-Boost & High & 40 \\
\hline Cuk & High & $50-60$ \\
\hline Flyback & High & Low \\
\hline Forward & High & $40-50$ \\
\hline Ful-Bridge & High & $40-50$ \\
\hline Uncontrolled Rectifier & Low & 60 \\
\hline Controlled Rectifier with & Low & $80<$ \\
\hline $\begin{array}{l}\text { Boost Converter } \\
\text { switched inductance }\end{array}$ & $70-75$ \\
\hline
\end{tabular}

\section{Converter Topologies In Energy Harvesting Modules}

The EH systems, apart from a few, generates AC output voltage. However, the WSN requires DC to function [21]. To facilitate this requirement of WSN, most of the EH modules have a rectifier integrated in their build. The array of transducers is connected in series topology and the combined input is applied to the rectifier. The output from the rectifier is filtered using a low pass filter.

The arrangement of a common EH system is as shown in figure 5. The DC voltage obtained at the load shown in figure 2 is fluctuating in nature and contains ripples. The efficiency of the EH module depends on the power made available to the module and the effective power obtained at the load [22-23,26]. This can be improved with the help of impedance matching between the sensor array and the rectifier circuit.

In $[15,17]$, the authors utilised a resistance-based approach for impedance-matching. This

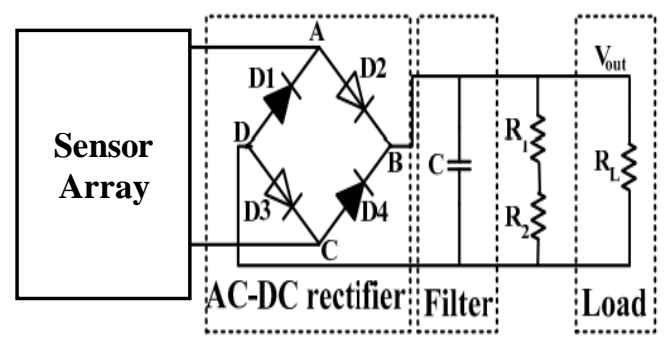

Fig 5. Common Energy Harvesting Circuit 
results in low power conversion efficiency. This problem was tackled using a synchronous switch harvesting technique on inductor (SSHI). The studies in $[28,29]$ proposed a 2-stage power management circuit (PMC) for rectifier and DC-DC regulator in EH. The PMC contained one rectifier, one buck-boost converter, a switching module, and a charging and discharging module for energy storage devices, as shown in figure 6.

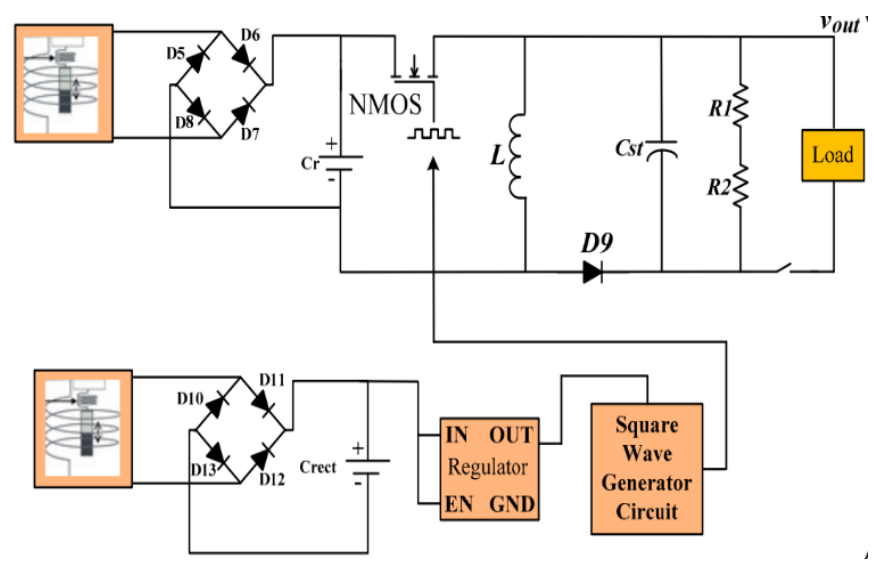

Fig 6.Power management circuit of $\mathrm{EH}$

A FWB rectifier is connected to the output of the transducer array[25]. The rectifiedoutput is provided to the DC-DC converter circuit as its input [24]. The pulse-width modulation (PWM) technique is called upon to control the switching. The control circuit contains a controller, a relational operator, a PWM modulator, and a sawtooth voltage generator. The output from the DC-DC converter is provided to the battery or the energy storage element [16]. The maindisadvantage of this model is that its inferior power conversion efficiency. This is due to the losses occurring at the converter due to its internal resistance. This can be addressed by reducing the total losses occurring during the converter operation.

In another workCao.X et al [20], have proposed the usage of boost converter in a closed loop topology and the switching control implemented with the help of PWM for feedback and feedforward control. Such an arrangement is as shown in fig 7. Wang et.al [27], proposed that rectifier output be directly given to the energy storage element when its value is sufficiently greater than forwarding voltage of the diode and the boost converter. Although efficient, this model is mathematically complex in nature and time consuming making it impractical for low power applications.

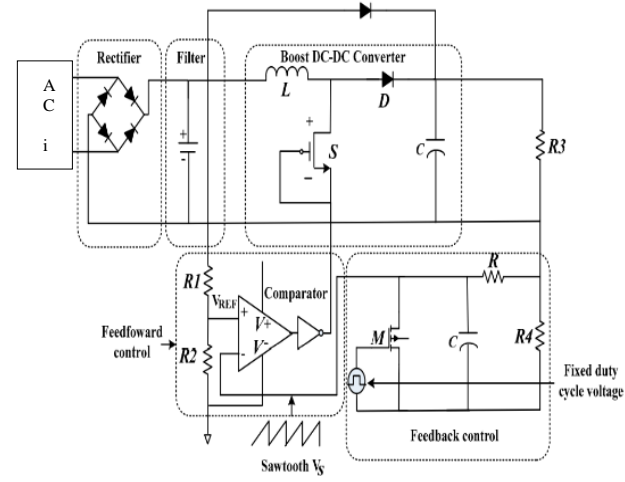

Fig 7. DC-DC Boost Converter based Energy harvester 
However, this can be solved using proper optimisation techniques to appropriately set the duty ratio of the NMOS switch.The authors in [28], [29] and [30] proposed SSHI circuit for harvesting energy from mechanical vibrations. The drawback for this approach is that opting for such a circuitry will result in low power conversion efficiency.

In RF-EH systems, rectifier-multiplier converters are sometimes preferred to individual rectifier-boost combinations.One such work, [12,13], authors suggested the usage of multiple diodes to build a n-stage rectifier-multiplier model. This topology has the inherent disadvantage of increased voltage drop due to larger number of diodes. The workaround for this problem is to utilise Schottky diodes in the place of normal diodes to reduce the total voltage drop due to the forward biasing of the diodes. The circuit for such an arrangement is as shown in the figure 8 .

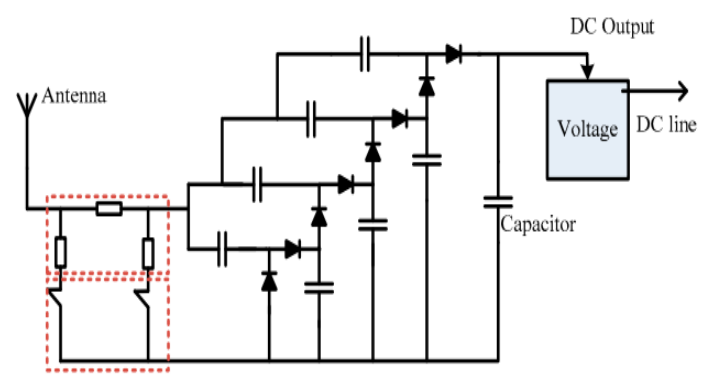

Fig 8.n-Stage Rectifier Circuit Model Used in RFEH System

\section{Conclusion}

This paper provides a review on EH systems. The emphasis is on the recent developments and the identification ofcurrent challenges. This study considers the application of EH systems in WSNs, building automation, self-powered devices and networks, remote health care units, wearable devices and low power electronic devices. It was clear from the conducted study that EH techniques will play an important role in improving the battery life or replacing the battery totally in these applications. When batteries are used to run the nodes of the sensors, it has a limited life. This reduces the reliability and performances of such systems.

$\mathrm{EH}$ techniques can overcome the aforementioned problems without adding to the burden of ever-growing power scarcity. Finally, harvesting energy from ambient energy resources offers several advantages, including the supply of potential energy to common low-power devices such as motion sensors. Furthermore, this review indicates the numerous types of power electronic topologies for the betterment of energy harvesting techniques. The study outlines a comprehensive survey of power electronic circuits, converters, controllers, and identified implementation in low-power applications to associate their significant contribution in the improvement.

The conduction and switching losses of an AC-DC converter are minimal, but the power factor and harmonic distortion are high. A DC-DC converter has a fast response time and high 
performance, but it suffers from high switching loss. Finally, various methods are recommended in this study to overcome the limitations of the controller,converter, and lowpower electronics devices for EMEH application.

\section{References}

[1] Sarker, M.R.; Julai, S.; Sabri, M.F.M.; Said, S.M.; Islam, M.; Tahir, M. "Review of piezoelectric energy harvesting system and application of optimization techniques to enhance the performance of the harvesting system. "Sens. Actuators A Phys. 2019, 300, 111634

[2] Jiao, D.; Ke, L.; Liu, S.; Chan, F.T. Optimal Energy-Delay in Energy Harvesting Wireless Sensor Networks with Interference Channels. Sensors 2019, 19, 785.

[3] Wang, H.; Li, W.; Xu, D.; Kan, J. A Hybrid Microenergy Storage System for Power Supply of Forest Wireless Sensor Nodes. Electronics 2019, 8, 1409.

[4] Li, Y.; Hamed, E.A.; Zhang, X.; Luna, D.; Lin, J.-S.; Liang, X.; Lee, I. Feasibility of Harvesting Solar Energy for Self-Powered Environmental Wireless Sensor Nodes. Electronics 2020, 9, 2058.

[5] Wang, Z.L.; Song, J. Piezoelectric nanogenerators based on zinc oxide nanowire arrays. Science 2006, 312, 242-246

[6] Sarker, M.R.; Mohamed, R.; Saad, M.H.M.; Tahir, M.; Hussain, A.; Mohamed, A. A Hybrid Optimization Approach for the Enhancement of Efficiency of a Piezoelectric Energy Harvesting System. Electronics 2021, 10, 75.

[7] Banerjee, S.; Bairagi, S.; Ali, S.W. A critical review on lead-free hybrid materials for next generation piezoelectric energy harvesting and conversion. Ceram. Int. 2021, 47, 16402-16421.

[8] A. Berzoy, E. Baethge, J. Restrepo and J. Viola, "Fuzzy Control System for Maximum Power Point Tracking in Solar Panels Based on DC-DC Converter PI Current Control," 2012 VI Andean Region International Conference, 2012, pp. 119-122.

[9] Shakeel, M.; Rehman, K.; Ahmad, S.; Amin, M.; Iqbal, N.; Khan, A. A low-cost printed organic thermoelectric generator for low-temperature energy harvesting. Renew. Energy 2020, 167, 853860.

[10] Falk, M.; Shleev, S. Hybrid dual-functioning electrodes for combined ambient energy harvesting and charge storage: Towards self-powered systems. Biosens. Bioelectron. 2019, 126, 275-291.

[11] Gholikhani, M.; Shirazi, S.Y.B.; Mabrouk, G.M.; Dessouky, S. Dual electromagnetic energy harvesting technology for sustainable transportation systems. Energy Convers. Manag. 2021, 230, 113804.

[12] Zhang, X.; Grajal, J.; López-Vallejo, M.; McVay, E.; Palacios, T. Opportunities and Challenges of Ambient Radio-Frequency Energy Harvesting. Joule 2020, 4, 1148-1152.

[13] Martins, G.C.; Mansano, A.L.; Stoopman, M.; Serdijn, W.A. Introduction to RF energy harvesting. In Wearable Sensors; Elsevier: Amsterdam, The Netherlands, 2021; pp. 311-336

[14] Asai, T.; Araki, Y.; Ikago, K. Energy harvesting potential of tuned inertial mass electromagnetic transducers. Mech. Syst. Signal. Process. 2017, 84, 659-672.

[15] Wang, J.; Dancy, A.J.; Ha, D.S. Vibration Energy Harvesting Circuit with Impedance Matching and Wake-up for Freight Railcars. In Proceedings of the IECON 2018 - 44th Annual Conference of the IEEE Industrial Electronics Society, Washington, DC, USA, 21-23 October 2018; pp. 19751979

[16] Cai, Q.; Zhu, S. Unified strategy for overall impedance optimization in vibration-based electromagnetic energy harvesters. Int. J. Mech. Sci. 2020, 165, 105198.

[17] Dancy, A.J.; Li, J.; Ha, D.S. Split-Capacitor Boost Converter Operating in Boundary Conduction Mode with Impedance Matching for Kinetic Energy Harvesting. In Proceedings of the 2020 IEEE 63rd International Midwest Symposium on Circuits and Systems (MWSCAS), Springfield, MA, USA, 9-12 August 2020; pp. 203-207

[18] Tehrani, S.Z.; Ranjbar, H.; Vial, P.; Premaratne, P. A New Efficient Power Management Interface for Hybrid ElectromagneticPiezoelectric Energy Harvesting System. In 16th International Conference on Information; Springer: Cham, Switzerland, 2019; Volume 800, pp. 537-542 
[19] tromagnetic Energy Harvesting with Enhanced Output Power. IEEE Trans. Power Electron. 2021, 1.

[20] Cao, X.; Chiang, W.-J.; King, Y.-C.; Lee, Y.-K. Electromagnetic Energy Harvesting Circuit with Feedforward and Feedback DC-DC PWM Boost Converter for Vibration Power Generator System. IEEE Trans. Power Electron. 2007, 22, 679-685.

[21] Wang, X.; Liang, X.; Wei, H. A study of electromagnetic vibration energy harvesters with different interface circuits. Mech. Syst. Signal. Process. 2015, 58-59, 376-398.

[22] Huang, T.-C.; Leu, Y.-G.; Huang, C.-W. Powering IoTs with a feedforward quasi universal boost converter energy harvester. Energy 2017, 133, 879-886.

[23] Huang, T.-C.; Leu, Y.-G.; Huang, C.-W. Powering IoTs with a feedforward quasi universal boost converter energy harvester. Energy 2017, 133, 879-886.

[24] Mitcheson, P.D.; Green, T.C.; Yeatman, E.M. Power processing circuits for electromagnetic, electrostatic and piezoelectric inertial energy scavengers. Microsyst. Technol. 2007, 13, 16291635.

[25] P, V.K.; Mahesh, P.G.S. An Efficient Direct AC-DC Converter for Low Voltage Energy Harvesting System. Int. J. Sci. Dev. Res. 2017, 2, 426-432

[26] Gao, L.; Lu, S.; Xie, W.; Chen, X.; Wu, L.; Wang, T.; Wang, A.; Yue, C.; Tong, D.; Lei, W.; et al. A self-powered and self-functional tracking system based on triboelectric-electromagnetic hybridized blue energy harvesting module. Nano Energy 2020, 72, 104684.

[27] Wang, H.; Tang, Y.; Khaligh, A. A Bridgeless Boost Rectifier for Low-Voltage Energy Harvesting Applications. IEEE Trans. Power Electron. 2013, 28, 5206-5214

[28] Wang, X.; Liang, X.; Wei, H. A study of electromagnetic vibration energy harvesters with different interface circuits. Mech. Syst. Signal. Process. 2015, 58-59, 376-398.

[29] Dong, X.; Huang, X. A Non-Resonant Type Electromagnetic Energy Harvester for Scavenging Vibration Energy. In Proceedings of the 2018 IEEE Sensors Applications Symposium (SAS), New Delhi, India, 28-31 October 2018; pp. 1-3.

[30] Arroyo, E.; Badel, A. Electromagnetic vibration energy harvesting device optimization by synchronous energy extraction. Sens. Actuators A Phys. 2011, 171, 266-273.

[31] GayathriMonicka, J \&Jamuna, V 2015, 'Hybrid Cascaded MLI topology using Ternary Voltage Progression Technique with Multicarrier Strategy', Journal of Electrical Engineering \&Technology (JEET) vol.10,pp.1610-1620,2015

[32] GayathriMonicka, J Jamuna.V, and Hemalatha.V, (2014), "A new approach for torque ripple minimization of PMBLDC motor drive" in the Lecture Notes in Electrical Engineering (LNEE), ISSN 1876-1100, Vol. 326, pp 285-294. 